\title{
Highlighted Papers
}

\section{Hydrophobic Amino Acids in the Hinge Region of the 5A Apolipoprotein Mimetic Peptide Are Essential for Promoting Cholesterol Efflux}

Apolipoprotein mimetic peptides are short amphipathic peptides that share the same biologic properties as full-length apolipoproteins and are being investigated as possible therapeutic agents for atherosclerosis. One such peptide is the $5 \mathrm{~A}$ peptide, which is a bihelical amphipathic peptide that contains the $18 \mathrm{~A}$ sequence in the first helix and a modified $18 \mathrm{~A}$ sequence containing 5 Ala substitutions in the second helix. Studies using a series of $5 \mathrm{~A}$ peptide analogs demonstrated that only peptides with hydrophobic amino acids in the hinge region were able to readily bind and solubilize phospholipid vesicles. These results were verified in vivo and provide a rationale for future design of therapeutic apolipoprotein mimetic peptides.

See article at J Pharmacol Exp Ther 2013, 344:50-58.

\section{Phosphoinositide-Dependent Kinase-1 Can Contribute to Hypertension}

Intermittent hypoxia increases blood pressure in rats along with increased endothelin-1 constrictor sensitivity in a protein kinase $\mathrm{C} \delta$ ( $\mathrm{PKC} \delta$ )-dependent manner. Because phosphoinositidedependent kinase-1 (PDK-1) regulates $\mathrm{PKC} \delta$, this study investigated the contribution of PDK-1 to hypoxia-induced hypertension. Inhibition of PDK-1 blunted endothelin-1 constriction in arteries from rats that had hypoxia-induced hypertension but not normal rats. In addition, treatment of rats with the PDK-1 inhibitor OSU-03012 [2-amino- $N$-\{4-[5-(2phenanthrenyl)-3-(trifluoromethyl)-1H-pyrazol-1-yl]-phenyl\}acetamide], lowered blood pressure in hypertensive rats but not in rats with normal blood pressure. The results suggest that intermittent hypoxia unmasks a role for PDK-1 in regulating endothelin-1 constrictor sensitivity and blood pressure, suggesting that PDK-1 may be effective treatment of some types of hypertension.

See article at J Pharmacol Exp Ther 2013 344:68-76.

\section{Screening for the Detection of G Protein- Coupled Receptor Heteromer Signaling}

Drugs targeting G protein-coupled receptors (GPCRs) make up more than $25 \%$ of all prescribed medications. The ability of GPCRs to form heteromers with unique signaling properties may be a new drug target. However, current in vitro assays are ill-equipped to detect heteromer-selective compounds. The present study describes the adaptation of an approach by using fusion proteins of GPCRs and chimeric G proteins to create an in vitro screening assay in which large numbers of compounds can be screened and only activated heteromers are detectable. This assay revealed that the $\delta$-opioid receptor agonist ADL5859 [ $N, N$-diethyl-4-(5-hydroxyspiro[chromene2,4'-piperidine]-4-yl)benzamide] has a 10 -fold higher potency on $\delta$-opioid receptors homomers than $\delta / \mu$-opioid receptor heteromers.

See article at $J$ Pharmacol Exp Ther 2013, 344:179-188.

\section{A Cationic Analog of Ceramide Induces Pancreatic Cancer Cell Death}

Treatment of pancreatic cancer that cannot be surgically resected currently relies on cytotoxic chemotherapy with gemcitabine. Resistance to gemcitabine is nearly universal and appears to involve defects in the mitochondrial apoptotic pathway. The sphingolipid ceramide is a critical mediator of apoptosis, and insufficient ceramide accumulation has been linked to gemcitabine resistance. LCL-124 [( $2 S, 3 S, 4 E)-2-$ $N$-[6'-(1' ${ }^{\prime \prime}$-pyridinium)-hexanoyl-sphingosine bromide] is a cationic ceramide that was effective in initiating apoptosis by causing mitochondrial depolarization in pancreatic cancer. LCL124 selectively accumulated and inhibited the growth of xenographs in vivo, and gemcitabine-resistant cells became more sensitive after treatment. These results suggest that this compound may be suited to overcome gemcitabine resistance.

See article at $J$ Pharmacol Exp Ther 2013, 344:167-178. 